\title{
Contents
}

Preface

by Jadwiga Daszyńska-Daszkiewicz

List of participants

\section{Session 0: Introduction}

Problems and prospects in stellar physics by M.-A. Dupret

\section{Session 1 A: DATA - Ground-based observations}

Photometric observations of pulsating stars (ground-based) by S. O. Kepler

Spectroscopic observations of pulsating stars by C. Aerts, M. Briquet, F. Carrier, et al.

Multi-wavelength photometric variation of PG $1605+072$ by S. Schuh, S. Dreizler, U. Heber, et. al

Radial-velocity observations of pulsating stars with

a new Poznań Spectroscopic Telescope by W. Dimitrow

Detecting pulsating variable stars from OGLE survey by I. Soszyński, A. Udalski, M. K. Szymański, et al.

Discovery of non-radial pulsations in the spectroscopic

binary Herbig Ae star RS Cha

by T. Böhm, W. Zima, C. Catala, et al.

A different approach to analyzing the Blazhko effect: the VSAA applied to RR Lyr by $\mathrm{K}$. Kolenberg, and S. Tsantilas

Regularities in the frequency spacings of Delta Scuti stars and the s-f Diagram by M. Breger, P. Lenz, and A. A. Pamyatnykh

\section{Session 1 B: DATA - Space missions}

Asteroseismology of sun-like stars with MOST

by D. B. Guenther, T. Kallinger, D. Huber, et al.

First asteroseismic results from CoRoT by E. Michel, A. Baglin, W. W. Weiss, et al.

First results on the Be stars observed with the CoRoT satellite by J. Gutiérrez-Soto, C. Neiner, A.-M. Hubert, et al.

\section{Session 2 A: MODES - Extracting eigenmode frequencies}

Extracting oscillation frequencies from sparse spectra: Fourier analysis by M. Jerzykiewicz

Application of the Trend Filtering Algorithm in the search for multiperiodic signals by G. Kovács, and G. Á. Bakos 
VSAA: A method of tracing variable frequencies in time series analysis

by $\mathrm{S}$. Tsantilas, and H. Rovithis-Livaniou

Asteroseismology of Red Giant stars

by N.J. Tarrant, W.J. Chaplin, Y.P. Elsworth, et al.

An application of Bayesian inference for solar-like pulsators

by $\mathrm{O}$. Benomar

\section{Session 2 B: MODES - Mode identification}

Pulsational mode identification from multi-colour photometry

- an observer's point of view by G. Handler

Constraints on angular numbers of pulsation modes from spectroscopy by J. H. Telting

High-resolution spectroscopy and mode identification in non-radially pulsating stars by K.R. Pollard, D.J. Wright, W. Zima, et al.

Spectroscopic mode identification of the $\delta$ Scuti star $4 \mathrm{CVn}$ by B. G. Castanheira, M. Breger, P. G. Beck, et al.

\section{Session 3 A: STARS - Convection}

Modeling stochastic excitation of acoustic modes in stars:

present status and perspectives

by R. Samadi, K. Belkacem, M.-J. Goupil, et al.

The effect of convection on pulsational stability

by $\mathrm{G}$. Houdek

Stochastic wave excitation in rotating stars by S. Mathis, K. Belkacem, and M.J. Goupil

The role of negative buoyancy in convective Cepheid models.

Double-mode pulsations revisited by R. Smolec

\section{Session 3 B: STARS - Opacity driving, levitation, opacity data}

The solar chemical composition by N. Grevesse

Input from opacity data in computation of pulsation instability by J. Montalbán, and A. Miglio

Radiative levitation and opacity driving: The potential of hot subdwarf pulsators for testing diffusion and other competing processes in stars by S. Charpinet, G. Fontaine, P. Brassard, and P. Chayer

The degenerate pulsators by $\mathrm{G}$. Fontaine, and P. Brassard

Long-term photometric monitoring of the hybrid subdwarf B pulsator HS $0702+6043$ by R. Lutz, S. Schuh, R. Silvotti, et al. 
Mode identification in rapidly pulsating subdwarf B stars from monochromatic amplitudeand phase variations

by S. K. Randall, V. van Grootel, G. Fontaine et al.

\section{Session 3 C: STARS - Effects of rotation on stellar structure and pulsation}

Effects of rotation on stellar structure: rotation induced mixing

by J.-P. Zahn

Pulsation in rapidly rotating stars

by U. Lee

Wave transport in differentially rotating stellar radiation zones by $\mathrm{S}$. Mathis

The asymptotic structure of the p-modes frequency spectrum in rapidly rotating stars by $\mathrm{F}$. Lignières, and $\mathrm{B}$. Georgeot

\section{Session 3 D: STARS - Effects of magnetic fields on stellar pulsation}

Theory of roAp stars

by $\mathrm{H}$. Shibahashi

Pulsation in the atmosphere of roAp stars

by $\mathrm{O}$. Kochukhov

Interferometric and seismic constraints on the roAp star $\alpha \mathrm{Cir}$ by I. M. Brandão, H. Bruntt, M. Cunha, and D. W. Kurtz

\section{Session 3 E: STARS - Oscillations and stellar models}

The impact of asteroseismology on the theory of stellar evolution

by C. S. Jeffery

Examples of seismic modelling

by A. A. Pamyatnykh

Asteroseismology of pre-main sequence stars

by K. Zwintz, D. Guenther, and T. Kallinger

Internal dynamics from asteroseismology for two

sdB pulsators in close binary systems

by V. Van Grootel, S. Charpinet, G. Fontaine, et al.

\section{Session 4: Future asteroseismic projects}

Kepler

by J. Christensen-Dalsgaard, T. Arentoft, T. M. Brown, et al.

BRITE-Constellation by W.W. Weiss

PLATO: PLAnetary Transits and Oscillations of stars by $C$. Aerts

Stellar Observations Network Group

by F. Grundahl, J. Christensen-Dalsgaard, T. Arentoft, et al. 
Conference summary: interpretations of asteroseismic data by J. Guzik

\section{Poster session}

Rho Puppis: some spectroscopic results or "The Taming of Rho Puppis" by V. Antoci, N. Nesvacil, and G. Handler

Solar-like stars as seen by CoRoT by R. A. García, T. Appourchaux, A. Baglin et al.

Preliminary seismic study of the $\gamma$ Doradus COROT target HD 49434 by M.-P. Bouabid, K. Uytterhoeven, A. Miglio, et al.

44 Tau: Examination of amplitude variability and combination frequencies by M. Breger, and P. Lenz

German Data Center for the Solar Dynamics Observatory:

A model for the PLATO mission?

by R. Burston, L. Gizon, Y. Saidi, and S.K. Solanki

Seismology of ZZ Ceti stars

by B. G. Castanheira, and S. O. Kepler

Contributions of different effects towards the light variations

in main sequence pulsators

by J. Daszyńska-Daszkiewicz

HARPS seismic data of the F type star HD 49933

revisited in the light of CoRoT results

by S. Deheuvels, E. Michel, B. Mosser, and C. Catala

Pulsating $B$ and Be stars in the Magellanic Clouds by P.D. Diago, J. Gutiérrez-Soto, J.Fabregat, et al.

Dipole modes of stellar oscillations

by G. Dogan, J. Christensen-Dalsgaard, and M. Takata

Asteroseismic models for the exoplanet host star HD 19994: A preliminary approach by M. E. Escobar, M. Soriano, S. Théado, and S. Vauclair

Radiative levitation: a likely explanation for pulsations

in the unique hot O subdwarf star SDSS J1600+0748

by G. Fontaine, P. Brassard, E.M. Green, et al.

New multisite observations of $\delta$ Scuti stars V624 Tauri and HD 23194

by L. Fox Machado, E. Michel, M. Chevreton, et al.

Pulsational analysis of the Herbig Ae star HD 140237 by $A$. Fumel, and T. Böhm

Is HD 163899 really a supergiant star? by M. Godart, M.A. Dupret, and A. Noels

Photometric observations of southern Blazhko Stars by E. Guggenberger, K. Kolenberg, and T. Medupe

Asteroseismology in the young open cluster NGC 3293 by G. Handler, T. Tuvikene, D. Lorenz, et al. 
Pulsations, chemical composition and multiplicity in main sequence $A$ - and F-type stars

by S. Hekker, Y. Frémat, P. Lampens, and P De Cat

Solar-like oscillations in red giants in the CoRoT exofield by S. Hekker, C. Barban, T. Kallinger, et al.

Orbital period analysis of some classical Algols with pulsating components by F. Soydugan, Y. Kaçar, E. Soydugan, et al.

Follow-up campaign of the Blazhko star RR Lyr by K. Kolenberg, N. D. Ulus, P.G. Beck, et al.

Spectroscopy of the sdB pulsator HS 2201+2610 by R. Kruspe, S. Schuh, R. Silvotti, and I. Traulsen

Frequency analysis of the $\delta$ Scuti type pulsations in the semi-detachedeclipsing binary CT Her by P. Lampens, A. Strigachev, S.-L. Kim, et al.

Photometric mode identification methods in eclipsing binaries by $\mathrm{O}$. Latkovic, and I. B. Biro

The oEA star TW Dra - a spectroscopic analysis by H. Lehmann, A. Tkachenko, V. Tsymbal, and D.E. Mkrtichian

Observational constraints on intrinsic mode amplitudes of $\delta$ Scuti pulsators by P. Lenz, J. Daszyńska-Daszkiewicz, A. A. Pamyatnykh, and M. Breger

The Algol-type eclipsing binary TZ Eridani:

BV photometry and search for pulsations and tertiary component by A.Liakos, B. Ulaș, K. Gazeas, and P. Niarchos

The ongoing campaign on the open cluster h Persei (NGC 869) by A. Majewska, A. Pigulski, and S.M. Ruciński

The near-contact system BF Velorum: New BVRI photometry and search for pulsations by V.N. Manimanis, C. Vamvatira-Nakou, and P.G. Niarchos

A Study of $\delta$ Scuti stars in open clusters NGC 1817 and NGC 7062 by J. Molenda-Żakowicz, S. Frandsen, and T. Arentoft

Nonradial modes in classical cepheids by P. Moskalik, and Z. Kołaczkowski

Multiperiodic RR Lyrae stars in $\omega$ Centauri by $\mathrm{P}$. Moskalik, and A. Olech

Main sequence pulsating stars in the galactic disk by A. Narwid, A. Pigulski, and Z. Kołaczkowski

The pulsations of the B5IVe star HD 181231 revealed by CoRoT by $\mathrm{C}$. Neiner, J. Gutierrez-Soto, Y. Frémat et al.

Atmospheric parameters of the $\beta$ Cephei star HD 167743 by E. Niemczura

Line profile variations in the bright subdwarf B star Balloon 090100001 by R. Oreiro, J. Telting, C. Aerts, and R. Østensen 
Prediciting amplitude variations of physical parameters from spectroscopic modelling of the pulsating sdBV Balloon 090100001

by R. Østensen, J.H. Telting, U. Heber, and C.S. Jeffery

Validity domain of a perturbative approach for rotational effects by R.-M. Ouazzani

Physical parameters determination of the RR Lyrae stars

RU Psc, SS Psc and TU UMa

by J. H. Peña, M. Chow, R. Peña, et al.

Perturbation analysis of sdO models

by C. Rodríguez-López, A. Moya, R. Garrido, et al.

Spectroscopy of pulsating stars at Poznan Spectroscopic Telescope data reduction and radial velocity measurements by A. Rożek, R. Baranowski, P. Bartczak, et al.

On the excited mode stability in the roAp star $\gamma$ Equ by M. Sachkov, T. Ryabchikova, M. Gruberbauer, and O. Kochukhov

A binary star with a $\delta$ Scuti component: EF Herculis by T. Șenyüz, and E. Soydugan

Seismic signatures of convective and/or helium cores by $\mathrm{M}$. Soriano, and S. Vauclair

Fourier analysis of gapped time-series by $\mathrm{T}$. Stahn, and L. Gizon

Radius determination from the large frequency separation by Dennis Stello, and the rest of the AsteroFLAG team

PMS $\delta$ Scuti stars in the region of Carina Nebula by $\mathrm{M}$. Stęślicki, and A. Pigulski

About the pulsational status of $\epsilon \mathrm{Oph}$ by D. Pricopi, and M. D. Suran

The driving mechanism of roAp stars : effects of global metallicity by S., Théado, M.-A., Dupret, and A., Noels

Spectroscopic solution for the oEA star RZ Cas using the SHELLSPEC code by A. Tkachenko, H. Lehmann, V. Tsymbal, and D. Mkrtichian

The preliminary results of the eclipsing binary system

EW Boo with a $\delta$ Scuti component by E. Soydugan M. Tüysüz, V. Bakıș, et al.

Detection of line-profile variations in high-resolution VLT/UVES spectroscopy of the subdwarf B pulsator PG 1336-018 (NY Virginis)

by M. Vučković, R. Østensen, J.H. Telting, et al.

Rotation and pulsation in g-mode main sequence pulsators by D. J. Wright, P. De Cat, K.R. Pollard, and W. Zima

Can opacity changes help to reproduce the hybrid star pulsations? by T. Zdravkov, and A. A. Pamyatnykh

FAMIAS - A userfriendly new software tool for the mode identification of photometric and spectroscopic times series 